\title{
Distributed Power Allocation for Parallel Relay Networks
}

\author{
Min Chen Semih Serbetli Aylin Yener \\ Electrical Engineering Department \\ The Pennsylvania State University \\ University Park, PA 16802 \\ muc163@psu.edu_serbetli@psu.edu_yener@ee.psu.edu
}

\begin{abstract}
We investigate power allocation strategies for distributed decode-and-forward (DF) parallel relay networks. We first propose a distributed decision mechanism for the relay nodes to make decisions on whether to forward the source data. Specifically, we identify the optimum distributed power allocation strategy that minimizes the total transmit power while providing a target Signal-to-Noise ratio (SNR) at the destination with a target outage probability. We also consider two simple distributed power allocation models, where the source does not contribute to the relay selection in the first model, and single relay is employed in the second model. Simulation results are presented to demonstrate the performance of the proposed distributed power allocation schemes, and the considerable power savings they provide with respect to random relay selection.
\end{abstract}

\section{INTRODUCTION}

Relay assisted transmission schemes are continuing to flourish [1]-[4] due to their potential of providing performance improvement in terms of outage behavior [2], achievable rate region [3]-[5], and error probability [6]-[8], without the need for physical antenna arrays. Power efficiency is a critical design consideration for wireless networks due to the limited transmission power of the relay nodes as well as the source. Optimum power allocation problem is studied upto-date in [8]-[11] for several relay transmission schemes. [10] and [11] discuss the optimum and near optimum power allocation schemes, respectively, for single-branch multi-hop relay networks. For multi-branch relay networks, [8] identifies the best relay selection strategy for amplify-and-forward (AF) relay networks, while [9] studies the efficient power allocation problem for both $\mathrm{AF}$ and $\mathrm{DF}$ parallel relay networks in wideband regime. However, to implement the optimum power allocation strategy, these centralized schemes require the feedback of channel state information (CSI) of all communication channels to the source for each channel realization, which may be infeasible in practice.

In this paper, we investigate distributed power allocation strategies for DF parallel relay networks for which the CSI is only partially known at the source and the relay nodes. We first propose a simple distributed decision mechanism for each relay node to individually make a decision on whether to forward the source data. Given such a decision scheme, we formulate the distributed power allocation problem, and identify the optimum solution based on the partially available
CSI at the source. We also consider two special cases with simpler implementation, namely the passive source model where the source does not contribute to the relay selection process, and the single relay model where only one relay node is selected. We term the extra power the distributed power allocation mechanism needs as compared to the optimum centralized power allocation mechanism the additional power expenditure, and observe the tradeoff between the outage probability and the additional power expenditure. We demonstrate that considerable power savings can be obtained by the proposed schemes with respect to random relay selection.

\section{System Model AND BACKGROUND}

We consider a relay network consisting of a sourcedestination pair and $N$ relay nodes employing decode-andforward. We assume that we have a time-slotted system. Let $f_{i}$ and $g_{i}$ denote the fading coefficients of the source-torelay and relay-to-destination channels for the $i$ th relay node, $i=1, \ldots, N$. The fading coefficient of the direct sourceto-destination link is denoted by $h$. We assume that each channel is flat fading, and $f_{i}, g_{i}$ and $h$ are all independent realizations of zero mean complex Gaussian random variables with variances $\sigma_{f_{i}}^{2}, \sigma_{g_{i}}^{2}$ and $\sigma_{h}^{2}$ per dimension, respectively. In the first time slot, the source broadcasts $X_{o}$ with power $P_{s}$. The destination observes $y_{d_{0}}$ :

$$
y_{d_{0}}=\sqrt{P_{s}} h X_{o}+z_{d_{0}}
$$

and the $i$ th relay observes $y_{r_{i}}$ :

$$
y_{r_{i}}=\sqrt{P_{s}} f_{i} X_{o}+z_{r_{i}} \quad \text { for } i=1, \ldots, N
$$

where $z_{d_{0}}$ and $\left\{z_{r_{i}}\right\}_{i=1}^{N}$ are the Additive White Gaussian Noise (AWGN) terms at the destination and the relays, respectively. Assume without loss of generality that they are of variance $1 / 2$ per dimension. The $i$ th relay is said to be reliable and can correctly decode $X_{o}$ when its received SNR, $S N R_{r_{i}}$ satisfies

$$
S N R_{r_{i}}=P_{s}\left|f_{i}\right|^{2} \geq S N R_{\text {target }}
$$

where $S N R_{\text {target }}$ is the given decodability constraint. In the second time slot, those relays who belong to the set of reliable relays, $A_{R}$, can forward the source data to the destination. Throughout this paper, we assume that the reliable relays regenerate source data with the same codebook used at the 
source. The signal received at the destination from the reliable relay $i$ is

$$
y_{d_{i}}=\sqrt{P_{i}} g_{i} X_{0}+z_{d_{i}}, \quad i \in A_{R}
$$

where $P_{i}$ is the transmit power of the $i$ th relay node, and $z_{d_{i}}$ is the normalized AWGN term at the $i$ th relay-to-destination channel. The destination combines signals received from relay nodes and the direct link with a maximum ratio combiner (MRC) and the resulting SNR at the destination is

$$
S N R_{d}=P_{s}|h|^{2}+\sum_{i \in A_{R}} P_{i}\left|g_{i}\right|^{2}
$$

We consider that the destination can correctly receive the source data when $S N R_{d} \geq S N R_{\text {target }}$. With this system model, we can pose the power allocation problem for regenerative DF relay networks as

$$
\begin{array}{cc}
\min _{P s,\left\{P_{i}\right\}} & P_{s}+\sum_{i \in A_{R}} P_{i} \\
\text { s. t. } & P_{s}|h|^{2}+\sum_{i \in A_{R}} P_{i}\left|g_{i}\right|^{2} \geq S N R_{\text {target }} \\
& P_{s}\left|f_{i}\right|^{2} \geq S N R_{\text {target }} \quad i \in A_{R}
\end{array}
$$

We note that the resulting power allocation strategy may prevent some reliable relays from participating simply by assigning zero power to those relays.

The optimum power allocation identified in [9] is also the optimum centralized power allocation strategy for regenerative DF relay networks. It can be expressed as

$$
\begin{aligned}
& P_{s}^{*}=\frac{S N R_{\text {target }}}{\left|f_{k^{*}}\right|^{2}} \\
& P_{i}^{*}=\left\{\begin{array}{l}
\left(\frac{S N R_{\text {target }}-|h|^{2} S N R_{\text {target }} /\left|f_{k^{*}}\right|^{2}}{\left|g_{k^{*}}\right|^{2}}\right)^{+}, i=k^{*} \\
0, \text { otherwise }
\end{array}\right. \\
& k^{*}=\arg \min _{\left\{k \in A_{E}\right\}}\left[\frac{1}{\left|f_{k}\right|^{2}}+\frac{1}{\left|g_{k}\right|^{2}}-\frac{|h|^{2}}{\left|f_{k}\right|^{2}\left|g_{k}\right|^{2}}\right]
\end{aligned}
$$

where $(\cdot)^{+}=\max (0, \cdot)$. The set $A_{E}$ denotes the set of relays such that the transmission through the relay is more power efficient than the direct transmission, i.e.,

$$
A_{E}=\left\{\left.i|| f_{i}\right|^{2} \geq|h|^{2} \cap\left|g_{i}\right|^{2} \geq|h|^{2}, i=1 . . N\right\}
$$

Since the source power is allocated to satisfy the SNR constraint with equality at the selected relay node, each relay node can decide whether it is the selected note by checking its received SNR.

We note that to implement the strategy given by (9)-(11), full CSI, i.e., $\left\{f_{i}, g_{i}\right\}_{i=1}^{N}$ and $h$, at the source node, and individual CSI, $\left\{f_{i}, g_{i}\right\}$, at relay node $i$ is needed. Although this scheme provides the most power efficient DF relay transmission strategy, its centralized nature, i.e., the fact that it requires the channel estimate of each link and the feedback of this information to the source, may render its implementation impractical. As such, distributed strategies are needed. In the following, we devise efficient distributed power allocation schemes.

\section{Distributed Power Allocation}

In this section, our aim is to find efficient distributed power allocation schemes using the partial CSI available at the source and the relays. In practice, it is feasible that, the source-torelay fading coefficients, $\left\{f_{i}\right\}_{i=1}^{N}$, can be obtained by training before actual data transmission at both the source and the relays, when each node operates in TDMA mode. However, it may be impractical to have $\left\{g_{i}\right\}_{i=1}^{N}$, the relay-to-destination coefficients, available at the source for each channel realization. Thus, we investigate distributed power allocation schemes when the source has the realizations $\left\{f_{i}\right\}_{i=1}^{N}$ and $h$, and only the statistics of $\left\{g_{i}\right\}$. The relay nodes are assumed to have their individual CSI, $f_{i}$ and $g_{i}$, for relay $i$.

We first derive a distributed decision mechanism with the model assumptions given above. Since the source has only the statistical description instead of $\left\{g_{i}\right\}_{i=1}^{N}$, the optimum centralized power allocation indicated by (9)-(11) cannot be implemented by the source. Also, while it is clear that for a fixed source power, the best strategy is transmitting through the reliable relay node that has the highest relay-to-destination channel gain, this mechanism requires a comparison of all $\left\{g_{i}\right\}_{i=1}^{N}$. The distributed relay decision should rely only on the individual CSI of the relays. Since each relay can easily determine whether it is a reliable relay by using its SNR value, i.e., its individual CSI, we propose that the $i$ th reliable relay decides it is the relay that has the largest channel gain to the destination when it satisfies

$$
\left|g_{i}\right|^{2} \geq \gamma
$$

where $\gamma$ is a given threshold value. It then forwards the decoded signal with sufficient power. That is, we have

$$
P_{i}^{*}=S N R_{\text {target }}^{\prime} /\left|g_{i}\right|^{2}
$$

where $S N R_{\text {target }}^{\prime}=\left(S N R_{\text {target }}-P_{s}|h|^{2}\right)^{+}$denotes the SNR contribution from the relay.

We note that such a distributed decision mechanism with any $\gamma>0$ results in a nonzero probability that none of the relay nodes satisfies (13), and hence a nonzero outage probability $\operatorname{Prob}\left(S N R_{d}<S N R_{\text {target }}\right)$. As such, the source should determine the optimum source power and the corresponding threshold $\gamma$ by considering the realizations of $\left\{f_{i}\right\}$ and the randomness in $\left\{g_{i}\right\}$, to meet a system given specification, i.e., an outage probability requirement.

Given the above decision strategy, we now investigate how the source should decide the value of its power and $\gamma$, to satisfy the target SNR, $S N R_{\text {target }}$ at the destination with a target outage probability, $\rho_{\text {target }}$. Since the relay transmit powers are random variables with known statistics from the source point of view, we consider the expected value of the relay transmit power

$$
E\left[P_{i}\left(\mathrm{~g}_{\mathrm{i}}, P_{s}, h, \gamma\right)\right]=\int_{\gamma}^{\infty} \frac{S N R_{\text {target }}^{\prime}}{2 \sigma_{g_{i}}^{2} X} \exp \left(\frac{-X}{2 \sigma_{g_{i}}^{2}}\right) d X
$$

where $X=\left|g_{\mathrm{i}}\right|^{2}$ and $\mathrm{g}_{\mathrm{i}}$ is complex Gaussian with $C\left(0, \sigma_{g_{i}}^{2}\right)$. The distributed power allocation problem can then be expressed as 


$$
\begin{array}{cc}
\min _{\gamma, P_{s}} & P_{s}\left(\left\{f_{i}\right\}, h,\left\{\sigma_{g_{i}}^{2}\right\}\right)+E\left[\sum_{i \in A_{R}\left(P_{s}\right)} P_{i}\left(\mathrm{~g}_{\mathrm{i}}, P_{s}, h, \gamma\right)\right] \\
\text { s. t. } & \operatorname{Prob}\left(S N R_{d} \leq S N R_{\text {target }}\right) \leq \rho_{\text {target }} \\
& P_{s}\left(\left\{f_{i}\right\}, h,\left\{\sigma_{g_{i}}^{2}\right\}\right)\left|f_{i}\right|^{2} \geq S N R_{\text {target }}, \quad i \in A_{R}
\end{array}
$$

The following theorem provides the optimum solution:

Theorem 1: The optimum source power, $P_{s}^{* *}$, can only be one of the $(N+1)$ discrete values in the set

$$
\left\{S N R_{\text {target }} /|h|^{2}, S N R_{\text {target }} /\left|f_{1}\right|^{2}, \ldots, S N R_{\text {target }} /\left|f_{N}\right|^{2}\right\}
$$

For each possible $P_{s}^{* *}$ value, there exist a corresponding reliable relay set $A_{R}^{* *}$, and a unique optimum $\gamma$ value, $\gamma^{* *}$.

Proof: Assume that $P_{s}=S N R_{\text {target }} /\left|f_{i}\right|^{2}$ and there exist a reliable relay set $A_{R}^{\dagger}$ containing $i$ relay nodes and a corresponding threshold value $\gamma^{\dagger}$. Then, the expected value of the total power is

$$
E\left[P_{\text {total }}\right]=P_{s}+\sum_{i \in A_{R}^{\dagger}} \int_{\gamma^{\dagger}}^{\infty} \frac{\left(S N R_{\text {target }}-P_{s}|h|^{2}\right)^{+}}{2 \sigma_{g_{i}}^{2} X} \exp \left(\frac{-X}{2 \sigma_{g_{i}}^{2}}\right) d X
$$

and the derivative of $E\left[P_{\text {total }}\right]$ with respect to $P_{s}$ is

$$
\frac{\partial E\left[P_{\text {total }}\right]}{\partial P_{s}}=1-|h|^{2} \sum_{i \in A_{R}^{\dagger}} \int_{\gamma^{\dagger}}^{\infty} \frac{1}{2 \sigma_{g_{i}}^{2} X} \exp \left(\frac{-X}{2 \sigma_{g_{i}}^{2}}\right) d X
$$

The direct transmission is more power efficient than the relay assisted transmission when the fading coefficient of the direct link is greater than all of the relay-to-destination links. That is when

$$
|h|^{2}>\frac{1}{\sum_{i \in A_{R}^{\dagger}} \int_{\gamma^{\dagger}}^{\infty} \frac{1}{2 \sigma_{g_{i}}^{2} X} \exp \left(-X / 2 \sigma_{g_{i}}^{2}\right) d X}
$$

In such a case, $P_{s}^{* *}=S N R_{\text {target }} /|h|^{2}$. On the other hand, the relay transmission is preferred when

$$
|h|^{2}<\frac{1}{\sum_{i \in A_{R}^{\dagger}} \int_{\gamma^{\dagger}}^{\infty} \frac{1}{2 \sigma_{g_{i}}^{2} X} \exp \left(-X / 2 \sigma_{g_{i}}^{2}\right) d X}
$$

We note that (23) implies $\frac{\partial E\left[P_{\text {total }}\right]}{\partial P_{s}}>0$, which means increasing $P_{s}$ beyond $S N R_{\text {target }} /\left|f_{i}\right|^{2}$ but within $S N R_{\text {target }} /\left|f_{i+1}\right|^{2}$ for $i=1, \ldots, N-1$ does not change $A_{R}^{\dagger}$ but increases the expected value of the total power $E\left[P_{\text {total }}\right]$. Similarly, any transmission with $P_{s}>S N R_{\text {target }} /\left|f_{N}\right|^{2}$ has larger $E\left[P_{\text {total }}\right]$ than the transmission with $P_{s}=$ $S N R_{\text {target }} /\left|f_{N}\right|^{2}$. Thus, the optimum source power $P_{s}^{* *}$ can be only one of the $(\mathrm{N}+1)$ discrete values in the set (19).

For $P_{s}=S N R_{\text {target }} /\left|f_{i}\right|^{2}$, one of the candidates of the optimum source power, and its corresponding reliable set $A_{R}^{\dagger}$, when $\gamma$ gets higher the expected value of the total power decreases, while the outage probability increases. Therefore, threshold $\gamma^{\dagger}$ should be chosen as the value that satisfies the outage probability with equality.

$$
\prod_{i \in A_{R}^{\dagger}}\left(1-\int_{\gamma^{\dagger}}^{\infty} \frac{1}{2 \sigma_{g_{i}}^{2}} \exp \left(-X / 2 \sigma_{g_{i}}^{2}\right) d X\right)=\rho_{\text {target }}
$$

The source should simply compare $(N+1)$ possible $E\left[P_{\text {total }}\left(P_{s}, \gamma\right)\right]$ values and decide the best $\left(P_{s}^{* *}, \gamma^{* *}\right)$ pair. Note that when the expected value of the total transmit power is higher than direct transmission, the source will prefer to transmit directly to the source.

The cost of the lack of full CSI at the source, i.e., the distributed decision mechanism, is an additional power expenditure. Let $P_{\text {total }}^{* *}\left(\left\{f_{i}\right\},\left\{\mathrm{g}_{\mathrm{i}}\right\}, h\right)$ and $P_{\text {total }}^{*}\left(\left\{f_{i}\right\},\left\{\mathrm{g}_{\mathrm{i}}\right\}, h\right)$ denote the total power of the proposed optimum distributed power allocation scheme and that of the optimum centralized allocation scheme given in (9)-(11), respectively. The expected value of the additional power expenditure is

$$
\begin{aligned}
& E\left[P_{\text {add }}\left(\left\{f_{i}\right\},\left\{\mathrm{g}_{\mathrm{i}}\right\}, h\right)\right] \\
= & E\left[P_{\text {total }}^{* *}\left(\left\{f_{i}\right\},\left\{\mathrm{g}_{\mathrm{i}}\right\}, h\right)\right]-E\left[P_{\text {total }}^{*}\left(\left\{f_{i}\right\},\left\{\mathrm{g}_{\mathrm{i}}\right\}, h\right)\right] \\
= & P_{s}^{* *}\left(\left\{f_{i}\right\},\left\{\mathrm{g}_{\mathrm{i}}\right\},\left\{\sigma_{g_{i}}^{2}\right\}\right)+\sum_{i \in A_{R}^{* *}} \int_{\gamma^{* *}}^{\infty} \frac{S N R_{\text {target }}^{\prime}}{2 \sigma_{g_{i}}^{2} X} \exp \left(\frac{-X}{2 \sigma_{g_{i}}^{2}}\right) d X \\
- & E\left[P_{\text {total }}^{*}\left(\left\{f_{i}\right\},\left\{\mathrm{g}_{\mathrm{i}}\right\}, h\right)\right]
\end{aligned}
$$

We observe that in (24) $\rho_{\text {target }}$ is an increasing function of $\gamma$, while in (26), $E\left[P_{a d d}\left(\left\{f_{i}\right\},\left\{g_{\mathrm{i}}\right\}, h\right)\right]$ is a decreasing function of $\gamma$. Thus, there exists a tradeoff between the outage probability and the additional power expenditure: reducing the target outage probability will require more additional power. While designing the power allocation strategy, a reasonable target outage probability should be chosen in accordance with this tradeoff.

\section{PAssive Source Model}

In practice, we may have situations where the source does not have the realizations of any of the channels, but has access only to the statistical descriptions of them. It may also be the case that the source may not be able to do computationally expensive operations, e.g. due to hardware constraints in sensor or RFID networks. We term such source nodes, passive. Considering these likely practical issues, in this section, we investigate the distributed power allocation for the passive source model.

Since each relay has its individual CSI, we can apply the same distributed decision mechanism as proposed in Section III. However, the passive source cannot optimize its power or $\gamma$; they should be fixed at appropriate values. Note that, different from Section III, in this case, we may end up having none of the nodes transmitting reliably to the destination. We next develop the criterion on how to choose the source power and $\gamma$ by considering the outage probability and the additional power expenditure jointly. The outage probability of the direct link is

$d_{\text {out }}=\operatorname{Prob}\left\{P_{s}|\mathrm{~h}|^{2}<S N R_{\text {target }}\right\}=1-\exp \left(-\frac{S N R_{\text {target }}}{P_{s} \cdot 2 \sigma_{h}^{2}}\right)$

For clarity of exposition, define $a_{i}, b_{i}$ and $c_{i}$ as

$$
\begin{gathered}
a_{i}=\operatorname{Prob}\left\{i \in A_{R}\right\}=\exp \left(-\frac{S N R_{\text {target }}}{P_{s} \cdot 2 \sigma_{f_{i}}^{2}}\right) \\
b_{i}=\operatorname{Prob}\left\{\left|\mathrm{g}_{\mathrm{i}}\right|^{2} \geq \gamma\right\}=\exp \left(-\frac{\gamma}{2 \sigma_{g_{i}}^{2}}\right) \\
c_{i}=\operatorname{Prob}\left\{i \in A_{C}\right\}=a_{i} \cdot b_{i}
\end{gathered}
$$

where $A_{C}$ denotes the set of relays that satisfy both (3) and 
(13). The overall outage probability becomes

$$
\rho_{\text {outage }}=\operatorname{Prob}\left\{A_{C}=\emptyset\right\} \cdot d_{\text {out }}=\prod_{i=1}^{N}\left[1-c_{i}\right] \cdot d_{\text {out }}
$$

While choosing $P_{s}$ and $\gamma$ to satisfy (31), we have two properties of $\left(P_{s}, \gamma\right)$ to be considered. The first one is

$$
\rho_{\text {outage }} \geq \prod_{i=1}^{N}\left[1-a_{i}\right] d_{\text {out }}
$$

where equality is satisfied when $\gamma=0$. Note that when $P_{s}$ is chosen close to the minimum value, the corresponding $\gamma$ is close to 0 , and most of the contribution comes from the relay transmission, resulting in many relays transmitting and hence extra relay power expenditure. Thus, an appropriate value for $\gamma$ should be chosen. Similarly, we have the second property as

$$
\rho_{\text {outage }} \geq \prod_{i=1}^{N}\left[1-b_{i}\right] d_{\text {out }}
$$

For a given $P_{s}$ value, $\gamma$ should be strictly less than some threshold to provide $\rho_{\text {target }}$.

Since relays employ the distributed decision mechanism, described in Section III, once again, a reasonable $\left(P_{s}, \gamma\right)$ pair should be chosen by considering both the tradeoff and their properties as in (31), (32) and (33).

\section{Single Relay Model}

The distributed power allocation schemes proposed up to this point in general result in multiple relays transmitting to the destination, causing additional power expenditure. In this section, we investigate the case where only one relay node selected by the source is allowed to transmit.

When relay $k$ is selected, the source transmit power should be $P_{s} \geq S N R_{\text {target }} /\left|f_{k}\right|^{2}$. For relay $k$ the most power efficient way is transmitting at $P_{k}\left(g_{k}\right)=S N R_{\text {target }}^{\prime} /\left|g_{k}\right|^{2}$ when $\left|g_{k}\right|^{2} \geq \tau_{k}$, and $\tau_{k}$ satisfies the outage probability constraint:

$$
\tau_{k}=-2{\sigma_{g_{k}}}^{2} \ln \left(1-\rho_{\text {target }}\right)
$$

The expected value of the transmit power of the relay node is

$E\left[P_{k}\right]=\int_{\tau}^{\infty} \frac{S N R_{\text {target }}^{\prime}}{X \cdot 2 \sigma_{g_{k}}{ }^{2}} \exp \left(\frac{-X}{2}\right) d X=\frac{S N R_{\text {target }}^{\prime} K(\tau)}{2 \sigma_{g_{k}}{ }^{2}}$

where $\tau=-2 \ln \left(1-\rho_{\text {target }}\right), K(\tau)=\int_{\tau}^{\infty} \frac{1}{X} \exp \left(\frac{-X}{2}\right) d X$, and $X=\left|g_{k}\right|^{2} / \sigma_{g_{k}}^{2}$. Observe that $E\left[P_{k}\right]$ is inversely proportional to the variance of the fading coefficient, ${\sigma_{g_{k}}}^{2}$.

We express the optimum power allocation problem as

$$
\begin{array}{cc}
\min _{P_{s}, k} & P_{s}+E\left[P_{k}\right] \\
\text { s. t. } & \operatorname{Prob}\left(S N R_{d} \leq S N R_{\text {target }}\right) \leq \rho_{\text {target }} \\
& P_{s}\left|f_{k}\right|^{2} \geq S N R_{\text {target }}
\end{array}
$$

Similar to Section III, Theorem 1 is applicable here, i.e., the optimum source power $P_{s}^{* *}$, has to be one of $N+1$ possibilities. Thus, the optimum solution can be expressed as

$$
\begin{aligned}
P_{s}^{* *} & =S N R_{\text {target }} /\left|f_{k^{* *}}\right|^{2} \\
k^{* *} & =\arg \min _{|h|^{2}<2 \sigma_{g_{k}}^{2} / K(\tau)} \frac{1}{\left|f_{k}\right|^{2}}+\frac{K(\tau)}{2 \sigma_{g_{k}}^{2}}\left(1-\frac{|h|^{2}}{\left|f_{k}\right|^{2}}\right)^{+}
\end{aligned}
$$

(39)-(40) results in only the relay selected by the source, $k^{* *}$, satisfying SNR target. Thus, each relay can decide whether it is the selected node by examining its own received SNR. From (34) and (35), we note that the tradeoff between the outage probability and the additional power expenditure is present in this scheme as well. We also note that the relay threshold $\tau_{k}$ is a scaled version of $\sigma_{g_{k}}^{2}$ for each relay $k$. The complexity for calculating the relay threshold at the source is significantly less compared to that of the optimum distributed power allocation scheme derived in Section III, making the model and the corresponding strategy given in this section attractive from a practical stand point.

\section{NUMERICAL RESULTS}

In this section, we present numerical results related to the performance of the proposed distributed power allocation schemes. We consider a relay network consisting of a source, a destination, and $N=15$ relay nodes that are distributed as shown in Figure 1. We assume the pass-loss model [12] on Rayleigh fading channels as in [2]. Thus, we have $\sigma_{f_{i}}^{2}=$ $C / d_{S R_{i}}^{\alpha}, \sigma_{g_{i}}^{2}=C / d_{R_{i} D}^{\alpha}$ and $\sigma_{h}^{2}=C / d_{S D}^{\alpha}$, where $d_{A B}$ is the distance between node $A$ and $B$, and $S, D$ and $R_{i}$ denote the source, the destination and the $i$ th relay node, respectively. The path-loss exponent is denoted by $\alpha$, and $C$ is a constant. The values $\alpha=3, C=7 \times 10^{-4}$, and $S N R_{\text {target }}=10$ are used throughout our simulations. The AWGN variances on all links are assumed to be $10^{-10}$.

Simulation results are presented to demonstrate the performance of the power allocation strategies. Specifically, we plot $E\left[P_{\text {total }}\right]$, the expected value of the total power expended versus $\rho_{\text {outage }}$, the target outage probability. Note that, for a fair comparison, we define that an outage occurs for the optimum centralized power allocation (OCPA) when the total transmit power is higher than a given power constraint.

In Figure 2, we observe that a substantial amount of power is saved by employing the distributed power allocation schemes, with respect to random relay selection, in which the source randomly selects one relay with equal probability. The power savings is more pronounced for low outage probability values. As expected, an additional power expenditure, which is the penalty of lack of full CSI, is introduced by all distributed allocation schemes. Again, as expected, the passive source model (PSM) and the single relay model (SRM) require more power than the optimum distributed power allocation (ODPA) scheme of Section III. However, observe that this additional required power is small. Hence PSM and SRM may be preferable for systems when computational complexity is at a premium.

Figure 2 also remarks that the performance of the system with a passive source is very much a function of the source power. For low outage probability values, a high source power value is favorable since it reduces the SNR contribution from the relay nodes, and hence the transmit power for the relay nodes. On the other hand, for high outage probability values, the source power becomes a lower bound for the total power, thus a low source power is preferred. 


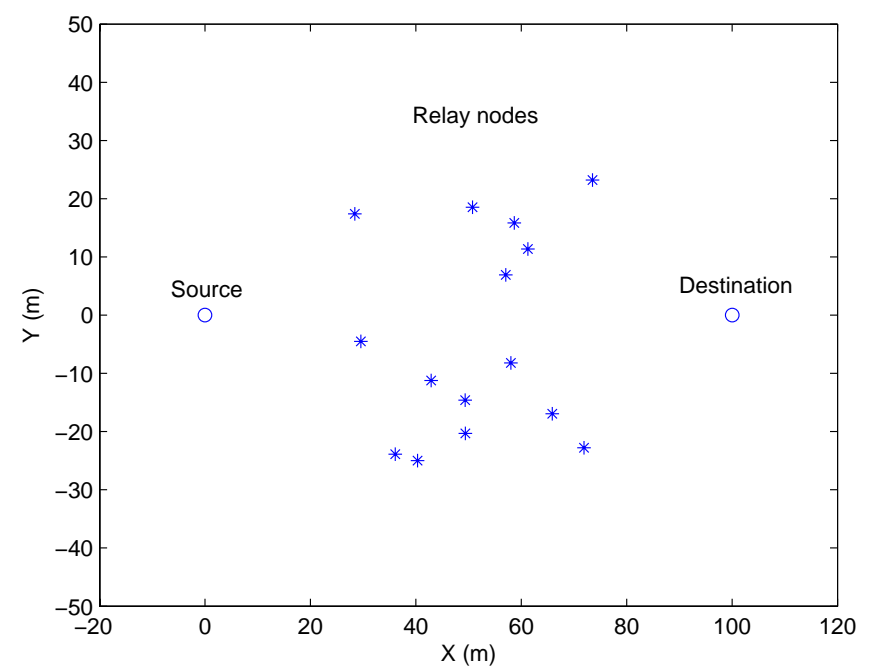

Figure 1. Relay network model

We also investigate the effect of the direct link on the performance. Figure 3 shows the effect of direct link SNR contribution on the passive source model and the single relay model. It is observed that a small amount of power savings is obtained when the direct link is considered. This amount vanishes as the quality of the direct link decreases. With this observation, when the direct link has a poor channel quality, the transmitting relay $i$ can forward the signal with power $S N R_{\text {target }} /\left|g_{i}\right|^{2}$ instead of $S N R_{\text {target }}^{\prime} /\left|g_{i}\right|^{2}$ without a significant performance loss. Employing such a strategy has the advantage that, the direct link, $h$, is not required at relays, reducing the amount of feedback from the destination.

\section{CONCLUSIONS}

In this paper, we have investigated distributed power allocation schemes and efficient relay selection mechanisms with partial CSI. We have observed that the proposed distributed methods perform close to the optimum centralized scheme. Our main result is that by using distributed power allocation and partial CSI, we can develop power efficient transmission schemes, reducing the amount of control traffic overhead.

\section{REFERENCES}

[1] T. M. Cover and A. A. El Gamal. Capacity theorems for the relay channel. IEEE Transactions on Information Theory, 25(5):572 - 584, September 1979.

[2] J. N. Laneman, D. N. C. Tse, and G. W. Wornell. Cooperative diversity in wireless networks: Efficient protocols and outage behavior. IEEE Transactions on Information Theory, 50(12):3062 - 3080, December 2004.

[3] A. Sendonaris, E. Erkip, and B. Aazhang. User cooperation diversitypart I: System description. IEEE Transactions on Communications, 51(11): 1927 - 1938, November 2003.

[4] A. Sendonaris, E. Erkip, and B. Aazhang. User cooperation diversitypart II: Implementation aspects and performance analysis. IEEE Transactions on Communications, 51(11):1939 - 1948, November 2003.

[5] P. Gupta and P. R. Kumar. Towards an information theory of large networks: an achievable rate region. IEEE Transactions on Information Theory, 49(8): 1877 - 1894, August 2003.

[6] A. Stefanov and E. Erkip. Cooperative coding for wireless networks. IEEE Transactions on Communications, 52(9):1470 - 1476, September 2004.

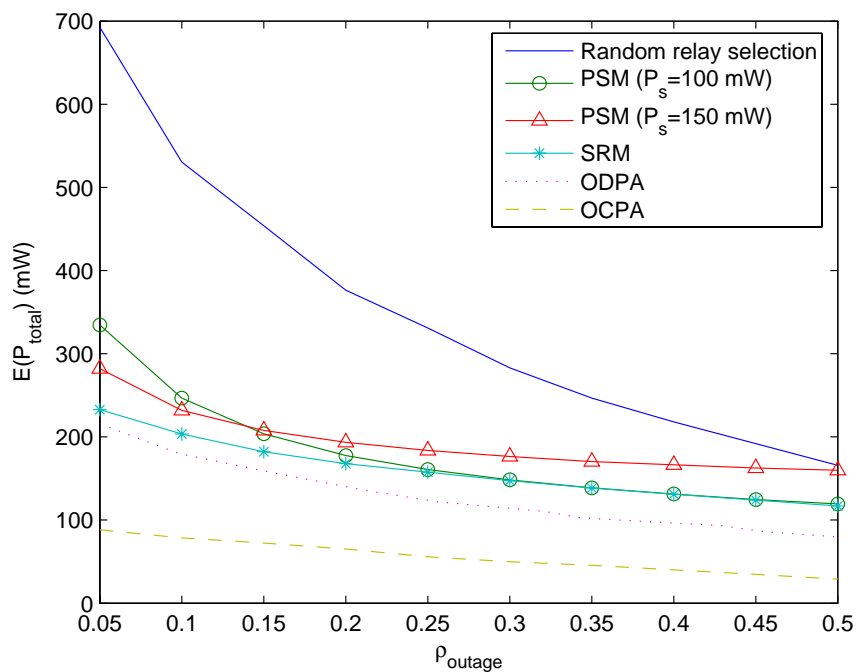

Figure 2. $E\left[P_{\text {total }}\right]$ vs $\rho_{\text {outage }}$ for different power allocation schemes.

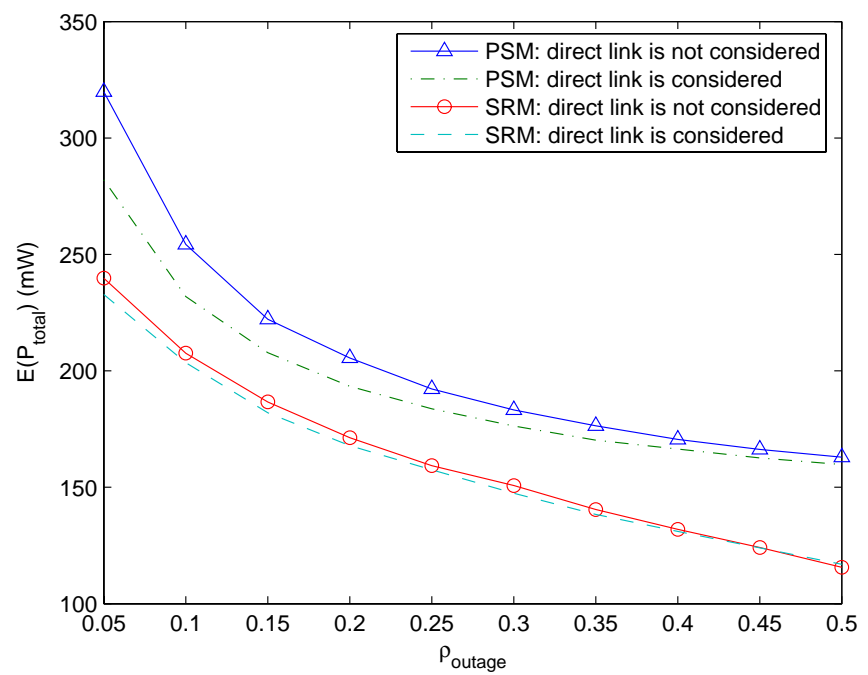

Figure 3. Effect of the direct link SNR contribution.

[7] M. Janani, A. Hedayat, T. Hunter, and A. Nosratinia. Coded cooperation in wireless communications: Space-time transmission and iterative decoding. IEEE Transactions on Signal Processing, 52(2):362 - 371, February 2004.

[8] A. Ribeiro, X. Cai, and G. B. Giannakis. Symbol error probabilities for general cooperative links. In IEEE International Conference on communications, ICC'04, pages 3369 - 3373, June 2004.

[9] I. Maric and R. Yates. Forwarding strategies for Gaussian parallel-relay networks. In Conference on Information Sciences and Systems, CISS'04, March 2004.

[10] M. O. Hasna and M.-S. Alouini. Optimal power allocation for relayed transmissions over Rayleigh-fading channels. IEEE Transactions on Wireless Communications, 3(6):1999 - 2004, November 2004.

[11] M. Dohler, A. Gkelias, and H. Aghvami. Resource allocation for FDMAbased regenerative multihop links. IEEE Transactions on Wireless Communications, 3(6):1989 - 1993, November 2004.

[12] T. S. Rappaport. Wireless Communications: Principles and practice. Prenice-Hall, 1996. 